\title{
Transition layer thickness at a fluid-porous interface
}

Cite as: Phys. Fluids 17, 057102 (2005); https://doi.org/10.1063/1.1894796

Submitted: 30 April 2004 . Accepted: 08 February 2005. Published Online: 13 April 2005

Afshin Goharzadeh, Arzhang Khalili, and Bo Barker Jørgensen

\section{ARTICLES YOU MAY BE INTERESTED IN}

Experimental analysis of the flow near the boundary of random porous media

Physics of Fluids 30, 047103 (2018); https://doi.org/10.1063/1.5021903

Velocity measurement of flow through a model three-dimensional porous medium

Physics of Fluids 18, 017105 (2006); https://doi.org/10.1063/1.2164847

Analysis of the Brinkman equation as a model for flow in porous media

The Physics of Fluids 30, 3329 (1987); https://doi.org/10.1063/1.866465

\section{Physics of Fluids GALLERY OF COVERS}




\title{
Transition layer thickness at a fluid-porous interface
}

\author{
Afshin Goharzadeh, Arzhang Khalili, ${ }^{a}$ and Bo Barker Jørgensen \\ Max Planck Institute for Marine Microbiology, Celsiusstrasse 1, 28359 Bremen, Germany
}

(Received 30 April 2004; accepted 8 February 2005; published online 13 April 2005)

\begin{abstract}
The length scale of the transition region between a porous layer and its overlying fluid layer is experimentally studied. The experimental setup consists of a rectangular channel, in which a fluid layer flows over a porous bed. Using particle image velocimetry and refractive index matching, two-dimensional velocity measurements in the interfacial region were performed. The thickness of this transition layer, defined by the height below the permeable interface up to which the velocity decreases to the Darcy scale, is measured and compared with the permeability and the matrix grain size. It was observed that the thickness of the transition zone, $\delta$, is of the order of the grain diameter, and hence, much larger than the square root of the permeability as predicted by previous theoretical studies. The Reynolds number and the fluid height over the porous substrate were found to affect the gradient of the horizontal velocity component at the interfacial region while the length scale of the transition layer remains approximately unchanged. The effect of the porous matrix type has been investigated by utilizing spherical glass beads as well as granulates. Scaling the measured velocities by the interfacial velocity near the uppermost solid matrix resulted in a unique velocity distribution in the case of monodisperse glass beads, hinting that the interfacial velocity represents a proper scaling factor. However, for polydisperse granulate material deviation from this behavior was observed. (C) 2005 American Institute of Physics. [DOI: 10.1063/1.1894796]
\end{abstract}

\section{INTRODUCTION}

When a porous layer is overlaid by a viscous fluid flow, a thin vertical transition zone between the layers is formed, which is responsible for the interfacial momentum and mass transfer. Understanding the mechanisms of interfacial transport has direct impact on many technical and natural phenomena. A very important example is given at permeable seabeds where a thin sediment-water interface, referred to as the benthic boundary layer (BBL), is known to have significant effects on the biology, geology, chemistry, and physics of aquatic systems. The lower portion of the BBL inside the sediment, called the Brinkman layer (or transition layer), has been identified to act as a transition zone, in which the viscous flow velocity in the water layer reduces drastically until it reaches an average velocity that can be predicted by Darcy's equation.

Although discussion of Brinkman layer thickness $\delta$ has been initiated by studies of heat and mass transfer in porous media, ${ }^{1-6}$ it has recently attracted considerable attention in marine microbiology because of its key role in understanding interfacial solute fluxes in benthic environments. ${ }^{7}$

To develop an expression for the permeability of packed beds, Brinkman ${ }^{1,8}$ matched the Stokes flow with the Darcy flow to obtain

$$
\nabla p=-\frac{\mu}{k} u+\tilde{\mu} \nabla^{2} u,
$$

with $p, k, u$, and $\mu$ denoting pressure, permeability, filter velocity, and dynamic viscosity, respectively, while the effec-

\footnotetext{
${ }^{\text {a) }}$ Author to whom correspondence should be addressed. Also at International University of Bremen, Campus Ring 1, 28759 Bremen, Germany. Electronic mail: akhalili@mpi-bremen.de
}

tive viscosity $\tilde{\mu}$ was given by $\tilde{\mu}=\mu[1+2.5(1-\varepsilon)]$ with $\varepsilon$ as porosity. Neale and Nader $^{2}$ used an extended Brinkman equation in order to predict the permeability of packed beds. One reason for success of the application of the Brinkman equation is that it predicts a boundary layer thickness $\delta$, which is on the order of $\sqrt{k}$, as suggested in many theoretical studies. ${ }^{6}$ However, a clear experimental identification of the Brinkman layer thickness has not been provided.

Recently, Gupte and Advani ${ }^{9}$ measured a local field flow at a permeable interface in a fibrous, preform-filled HeleShaw cell using laser Doppler velocimetry, and concluded that the boundary layer zone was on the order of the channel depth, and thereby larger than the square root of the permeability. However, because of the specific nature of their experimental setup and restrictions provided by the Hele-Shaw cell, their conclusion cannot be considered as universal and valid for transition layer thickness at fluid-porous interfaces. Therefore, the question of the extent of the Brinkman layer still remains. This can be partially related to the difficulties in two- or three-dimensional measurements and flow visualizations within porous layers. Although it is possible to visualize the fluid flow inside a porous medium via positron emission tomography (PET) ${ }^{10}$ due to the relatively low resolution of PET quantitative flow measurements are difficult to perform. The refractive index matching technique (RIM) has made flow measurements in porous media possible. This technique was performed in the past by matching the refractive indices of the fluid and solid boundaries of wall-bounded flows. ${ }^{11}$

Meanwhile, several researchers have made efforts to measure the flow field inside porous media by means of refractive index matching and flow visualization techniques. ${ }^{12-14}$ 


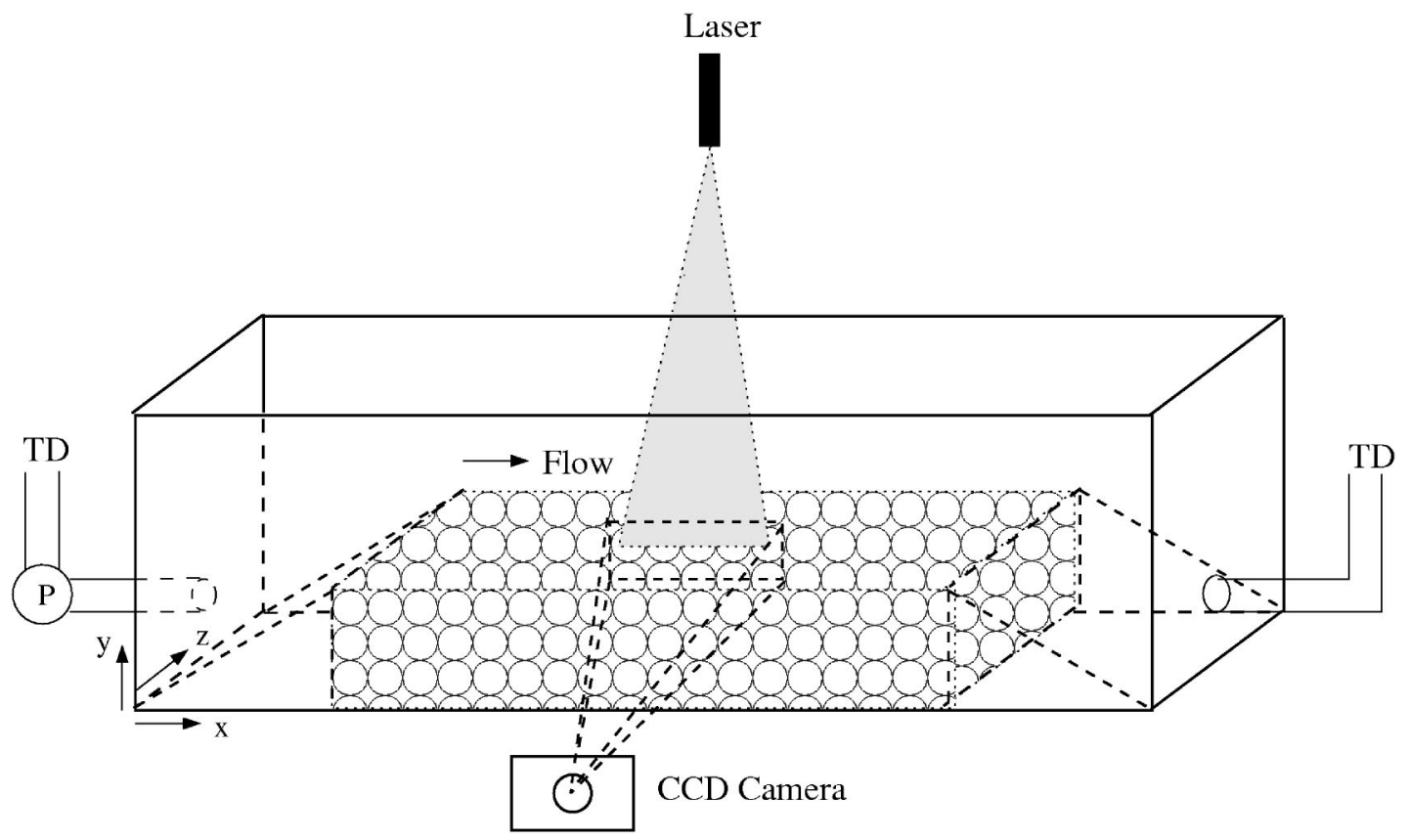

FIG. 1. Experimental setup. The pump $P$ generates a recirculating flow through and above the porous layer.

In this paper, we report the results of a nonintrusive optical approach using RIM and PIV (particle image velocimetry) in order to observe the field flow at a permeable interface and estimate the transition layer thickness. For this purpose, the solid matrix has been replaced by transparent glass beads or granulates, and the fluid by a mixture of two silicon oils, which has the same refractive index as that of the glasses. Using PIV, the two-dimensional (2D) velocity fields are measured for ten different sections along the permeable interface, and are averaged to obtain a single vertical velocity profile, which serves as the base for prediction of the transition layer thickness. In what follows, the experimental setup and details of the RIM and PIV system are explained first in Sec. II. The results of this study are presented in Sec. III, which contains measurements of the velocity field and the velocity profiles in the vicinity of the fluid-porous interface. Furthermore, it details the influence of medium permeability, Reynolds number, and the fluid height above the interface on the velocity profiles. Finally, in Sec. IV, some discussions on the length scale of the transition layer and the role of the interfacial velocity as a scaling parameter are presented.

\section{EXPERIMENTAL CONDITIONS AND MEASUREMENT TECHNIQUES}

\section{A. Experimental setup}

The experimental setup consists of a rectangular horizontal open channel of size $50 \times 10 \times 5 \mathrm{~cm}^{3}$ with sidewalls made of transparent Duran glass (see Fig. 1).

The central region of the extent $34 \times 4 \times 5 \mathrm{~cm}^{3}$ is filled by a random packing of a porous sample. Experiments were conducted using five different types of transparent borosilicate monodisperse glass beads and polydisperse granulates with physical properties reported in Table I. The porosity and permeability were measured separately by laboratory experi- ments. Porosity measurements were carried out using highprecision weighing equipment and were repeated five times to obtain an average value. For measurement of permeabilities of different samples, a technique similar to that described by Givler and Altobelli ${ }^{15}$ was used except that here oil was used instead of water. The permeability measurements were also repeated five times to obtain an average value.

The porous material is embedded between two 5 $\times 5 \mathrm{~cm}^{2}$ filters. The upper surface of the porous bed is flattened with a solid object to ensure a horizontal interface. The flume is then filled with the silicon oils mixed at the ratio reported in the following section. The height of the porous layer is constant for all experiments $\left(H_{p}=4 \mathrm{~cm}\right)$. A uniform pressure gradient is maintained in the longitudinal direction of the channel by a pump with an approximate maximal flow capacity of $Q=5 l \mathrm{~min}^{-1}$. In the central region of the channel, the flow over the porous surface is laminar and characterized by a constant fluid-based Reynolds number

$$
\operatorname{Re}_{f}=\frac{u_{\max } H_{f}}{\nu},
$$

where $u_{\max }$ is the maximum flow velocity at the fluid surface in the $x$ direction, $H_{f}$ the height of the fluid layer over the

TABLE I. Specifications and physical features of porous material: glass beads (GB) and granulates (GL).

\begin{tabular}{clcc}
\hline \hline Samples & \multicolumn{1}{c}{$d(\mathrm{~cm})$} & \multicolumn{1}{c}{$k\left(\mathrm{~m}^{2}\right)$} & $\varepsilon$ \\
\hline GL1 & $0.05-0.2$ & $5.2 \times 10^{-10} \pm 8.8 \times 10^{-12}$ & $0.43 \pm 0.01$ \\
GL2 & $0.2-0.35$ & $3.9 \times 10^{-9} \pm 6.7 \times 10^{-11}$ & $0.41 \pm 0.01$ \\
GB1 & 0.25 & $5.2 \times 10^{-9} \pm 8.4 \times 10^{-11}$ & $0.38 \pm 0.01$ \\
GB2 & 0.47 & $1.1 \times 10^{-8} \pm 3.1 \times 10^{-10}$ & $0.41 \pm 0.01$ \\
GB3 & 0.65 & $1.3 \times 10^{-8} \pm 6.9 \times 10^{-10}$ & $0.41 \pm 0.01$ \\
\hline \hline
\end{tabular}


TABLE II. Definition of the Reynolds number for different characteristic lengths $L$ and velocities $U$.

\begin{tabular}{ccc}
\hline \hline$L$ & $U$ & \multicolumn{1}{c}{$\mathrm{Re}$} \\
\hline$h_{f}$ & $u_{\text {max }}$ & $\operatorname{Re}_{f}=21$ \\
$d$ & $u_{\text {int }}$ & $\operatorname{Re}_{\text {int }}=0.45$ \\
$\sqrt{k}$ & $u_{D}$ & $\operatorname{Re}_{k}=0.0006$ \\
$d$ & $u_{D}$ & $\operatorname{Re}_{p}=0.033$ \\
\hline \hline
\end{tabular}

porous matrix, and $\nu$ the kinematic viscosity of the fluid. The maximum velocity is controlled using a valve in order to set a different Reynolds number. The kinematic viscosity $\nu$ was measured by an Ubbelohde capillary viscometer (Schott $52520 / \mathrm{II})$ for the silicon oil mixture as $\nu=42.5$ $\times 10^{-6} \mathrm{~m}^{2} / \mathrm{s}$. The fluid is maintained at a constant temperature $T=23{ }^{\circ} \mathrm{C}$ using a recirculating thermal device.

Although the flow in our geometry can be described uniquely by Eq. (2), it should be noted that this Reynolds number is based on the maximum velocity in the fluid layer, and hence, is not characteristic for the velocity in the entire domain. However, in order to characterize the flow in the porous layer, it has been customary $\left(\right.$ Ward $^{16}$ ) to introduce a "superficial" Reynolds number based on the permeability of the porous medium $k$ and the characteristic Darcy velocity $u_{D}$ given by

$$
\operatorname{Re}_{k}=\frac{u_{D} \sqrt{k}}{\nu} \text {. }
$$

The pore-scale Reynolds number has also been calculated in previous works (see, for example, the works by Kaviany, ${ }^{6}$ Nield and Bejan ${ }^{17}$ ) based on the average grain size $d$ and has been referred to as the "particle" Reynolds number given by

$$
\operatorname{Re}_{p}=\frac{u_{D} d}{\nu} .
$$

Likewise, a characteristic Reynolds number for the interfacial region would be based on the interfacial velocity $u_{\text {int }}$ and the average grain diameter, and can be given by

$$
\operatorname{Re}_{\text {int }}=\frac{u_{\text {int }} d}{\nu} \text {. }
$$

Using previous equations we have calculated the characteristic Reynolds numbers associated with flow inside the porous media (see Table II). As shown in Table II, all of the Reynolds numbers, defined for porous media, are much below 1.

\section{B. Visualization techniques}

To allow optical access to the interior of a medium that contains a solid matrix, sediment was replaced by transparent glass (beads and granulates). After a detailed survey of the chemistry literature, two suitable silicon oils (Dow Corning 550 and 556) were found, and mixed in a certain volume fraction, respectively, $32.2 \%$ and $67.8 \%$ at $T=23{ }^{\circ} \mathrm{C}$ to provide the same refractive index in fluid as well as borosilicate glass. The physical properties of both silicon oils, the boro-
TABLE III. Properties of silicon oils, mixture oil, and borosilicate.

\begin{tabular}{cccc}
\hline \hline & $\rho\left(\times 10^{3} \mathrm{~kg} / \mathrm{m}^{3}\right)$ & $\nu\left(\times 10^{-6} \mathrm{~m}^{2} / \mathrm{s}\right)$ & $n$ \\
\hline DC 550 & 1.068 & 125 & 1.4935 \\
DC 556 & 0.980 & 22.5 & 1.4600 \\
Mixture & 1.006 & 42.5 & 1.4736 \\
Borosilicate glass & 2.23 & $\ldots$ & 1.471 \\
\hline \hline
\end{tabular}

silicate glass beads and granulates are given in Table III. As shown in Table III, the mixture of two silicon oils provides a suitable refractive index $(n=1.4736)$ corresponding to that of borosilicate glass beads and granulates $(n=1.471)$ used.

The flow in the fluid as well as in the porous layer was examined using PIV. For this purpose, polyamide particles were added to the fluid as seeding tracers. In order to obtain optimal visualizations different tracer sizes were chosen depending on the size of the glass beads or granulates (see Table IV).

To illuminate the central cross section of the flume, a diode laser was mounted above the channel. A chargecoupled device (CCD) camera was installed perpendicular to the plane of the laser sheet to record the particle motion in the field of view, as illustrated in Fig. 1. Using a mobile mechanical device the CCD and laser were moved simultaneously in order to record multiple interfacial images along the channel.

Full-frame images of $512 \times 512$ pixels were acquired and transferred to a computer via a frame grabber. The measurements focused on the interface region covering portions of the fluid layer and the porous layer $25 \mathrm{~cm}$ downstream from the inlet of the flow. Note that the $x$ axis denotes the horizontal flow direction and $y$ axis is the vertical direction.

An example of a PIV image is shown in Fig. 2(a), which demonstrates an interfacial region after using refractive index matching. The flow in the fluid layer was observed with a large field of view $\left(4.5 \times 4.5 \mathrm{~cm}^{2}\right)$. The single-image measurements were focused in the central region of the channel $(x \simeq 25 \mathrm{~cm})$, whereas the multiple images were obtained by moving the mobile mechanical device to cover a horizontal length of $20<x<30 \mathrm{~cm}$. The time between each couple of images was $322 \mathrm{~ms}$. The exposure laser pulse time and pulse separation time were, respectively, $t_{e}=1 \mathrm{~ms}$ and $\Delta t_{s}=10 \mathrm{~ms}$. The upper part of Fig. 2(a) is the fluid region, whereas the lower part is the porous bed of glass beads. The tiny bright spots are tracer particles and the dark round areas show the locations of the glass beads. Note that all the beads shown are of the same size, and their different appearance in the figure is due to the fact that the laser sheet sections the spheres randomly and, hence, produces cross sections of dif-

TABLE IV. Diameter of the polyamide particle tracers used for different glass beads and granulates in the PIV measurements.

\begin{tabular}{llllll}
\hline \hline Diameter & GL1 & GL2 & GB1 & GB2 & GB3 \\
\hline Borosilicate glass $\left(\times 10^{-3} \mathrm{~m}\right)$ & $0.5-2$ & $2-3.5$ & 2.5 & 4.7 & 6.5 \\
Polyamide tracer $\left(\times 10^{-6} \mathrm{~m}\right)$ & 5 & 10 & 5 & 10 & 22 \\
\hline \hline
\end{tabular}




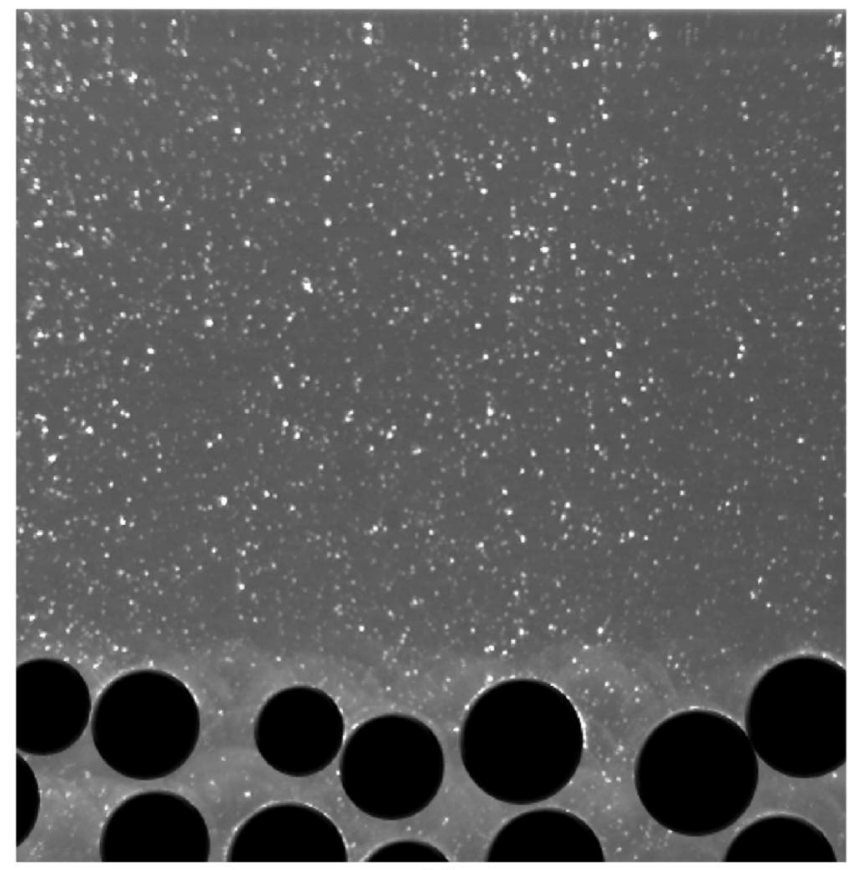

(a)

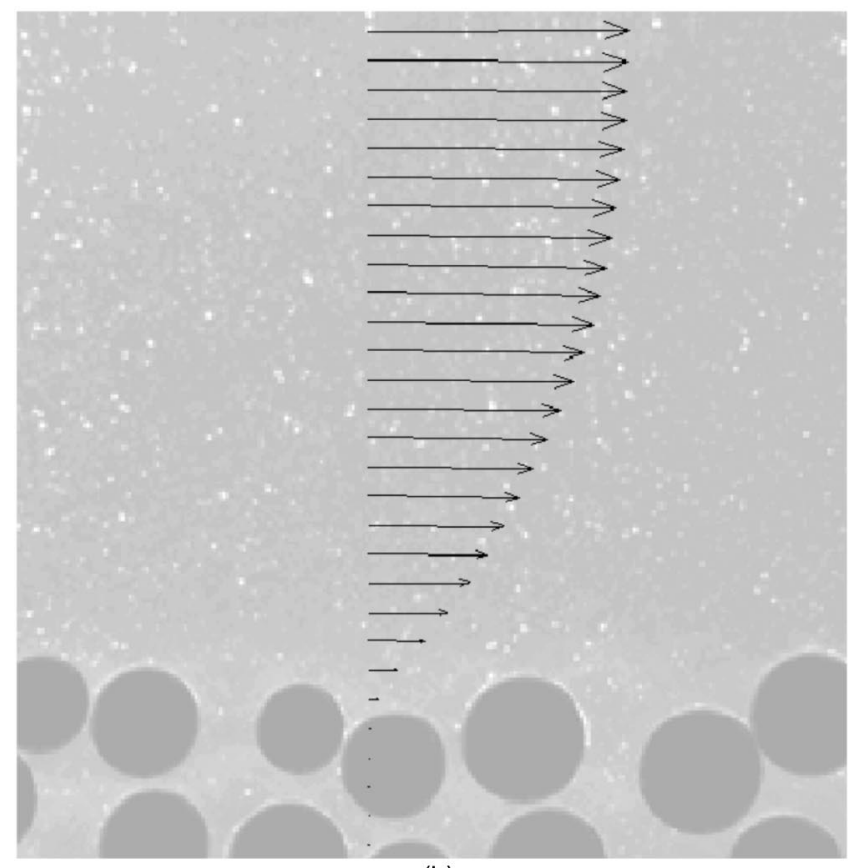

(b)

FIG. 2. (a) Example of PIV image in $x-y$ plane with a large field of view $\left(4.5 \times 4.5 \mathrm{~cm}^{2}\right)$ and $(\mathrm{b})$ the corresponding vertical averaged profile of horizontal velocity component $\left(\mathrm{GB} 3, H_{f}=4 \mathrm{~cm}, \mathrm{Re}_{f}=21\right)$.

ferent sizes. For the same reason, the beads seem as if they would not always touch their neighbors. A typical velocity profile of the horizontal component is plotted in Fig. 2(b). The maximum horizontal velocity component $u_{\max }$ appears at the upper part of the flow near the free surface. The horizontal velocity component is constant in the vicinity of the free surface and decreases continuously until it reaches the porous bed.

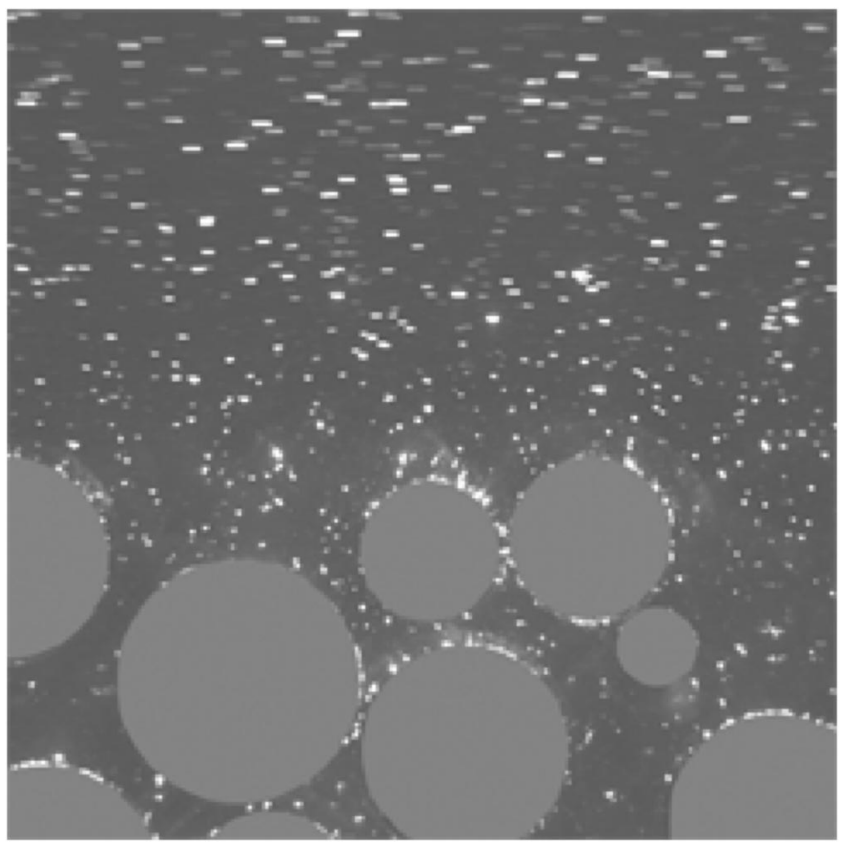

FIG. 3. Example of PIV image in $x-y$ plane with a large field of view $\left(2.2 \times 2.2 \mathrm{~cm}^{2}\right)$ for GB3.

\section{RESULTS}

\section{A. Velocity field at the fluid-porous interface}

To observe the flow near the interface at pore-scale, a lens with a focusing length of $f=5 \mathrm{~cm}$ was employed and connected to an extension tube with a total length of $2 \mathrm{~cm}$ between the CCD camera and the lens. In this way, a field of view of $2.2 \times 2.2 \mathrm{~cm}^{2}$ was obtained (Fig. 3). The time between each couple of images is $322 \mathrm{~ms}$. As far as the exposure laser pulse time $t_{e}$ and the pulse separation time step $\Delta t_{s}$ are concerned, it should be noted that a single pair of $\left(t_{e}, \Delta t_{s}\right)$ would fail to provide a correct overall velocity field due to drastic changes of velocity scale on either sides of the interface. Hence, two sets of optimal exposure laser pulse time and pulse separation time steps were necessary, namely $\left(t_{e}\right.$ $\left.=9 \mathrm{~ms}, \Delta t_{s}=10 \mathrm{~ms}\right)$ for the fluid region and $\left(t_{e}=50 \mathrm{~ms}, \Delta t_{s}\right.$ $=100 \mathrm{~ms}$ ) for the porous region.

To quantify the observed flow, we measured the velocity vector field in the $(x, y)$ plane by postprocessing of the particle images (Fig. 4). Velocities in the fluid layer $u_{f}$ are much higher than those inside the porous medium $u_{m}$. In a single vector plot covering the entire interface region, the velocity vectors in the porous region would, therefore, not be visible because of their very small magnitudes compared to vectors in the fluid layer. Hence, the image (Fig. 4) has been split in two parts separated at $y=-0.2 \mathrm{~cm}$, in which velocity fields are presented with two different magnification factors. In the upper region, far from the permeable interface, fluid particles have rectilinear trajectories, whereas in the lower region, they change directions and indicate disturbances due to the rough interface. The location of the permeable interface was defined by the ordinate of the uppermost solid (granulates or glass beads) in all images and is shown as $y=0$.

As the fluid particles approach the interface, their veloci- 


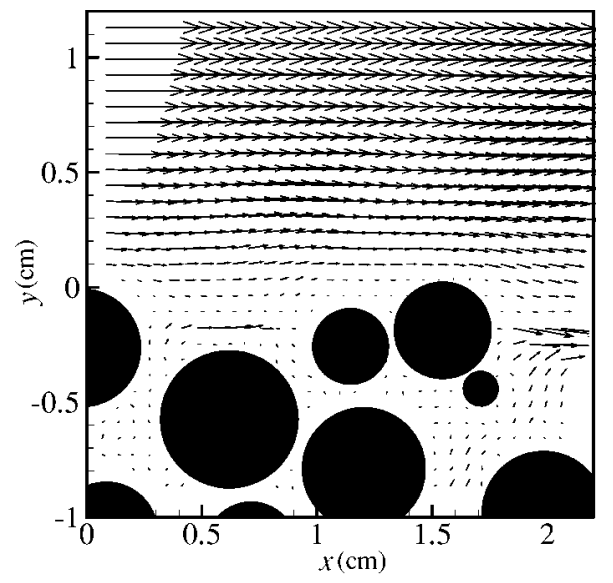

FIG. 4. Velocity vector plot covering the interfacial region.

ties decrease. In deeper layers inside the porous medium, the magnitude of the horizontal velocities drops drastically to $2.8 \times 10^{-3} \mathrm{~cm} / \mathrm{s}$ (at $y=-0.31 \mathrm{~cm}$ ), and is two orders of magnitude less than the velocity in the fluid region (4.4 $\times 10^{-1} \mathrm{~cm} / \mathrm{s}$ at $y=0.1 \mathrm{~cm}$ ).

\section{B. Velocity profiles}

To start with the analysis, the horizontal velocity components are calculated by averaging the obtained velocity over the fluid volume $V_{f}$ as well as over a volume that contains both fluid and solid $\left(V_{m}\right)$. The corresponding velocity components are denoted by $U$ and $u$, respectively. The average is obtained using ten neighboring sections with a small field of view $\left(2.2 \times 2.2 \mathrm{~cm}^{2}\right)$ along the $x$ coordinate separated by a regular distance of $1 \mathrm{~cm}$. The horizontal velocity profiles $U$ and $u$ are plotted in Fig. 5.

The maximum horizontal velocity set near the free surface was $u_{\max }=2.25 \mathrm{~cm} / \mathrm{s}$ (at $\left.y=3.9 \mathrm{~cm}\right)$. As can be seen from Fig. 5(a), in the fluid region the profiles of $U$ and $u$ are identical. The deviation of $U$ from $u$ appears inside the porous region [Fig. 5(b)], and can be seen more clearly in Fig. 5(c). In addition, it should be noted that the decrease in both velocity profiles is continuous within the interfacial region. The magnitude of the interfacial velocity $u_{\text {int }}$ at $y=0$ was 0.3 $\mathrm{cm} / \mathrm{s}$. The averaged horizontal velocity components decrease drastically within a rather small layer. This layer, below the permeable interface, represents a transition zone and is often referred to as the Brinkman layer.

The approximate vertical extent of the transition layer may now be obtained by detailed analysis of Figs. 5(b) and 5(c). Departing from the experimental values obtained, two different criteria have been employed to address the thickness of the transition layer. The first criterion is based on a statistical test and the second one assumes boundary layer thickness definitions.

As can be seen from the measurements (see Table V), below the interface there exists a critical point ( $y$ $\left.=-0.66 \mathrm{~cm}, u=1.7 \times 10^{-4} \mathrm{~cm} / \mathrm{s}\right)$. Above this point the velocity increases monotonically, whereas below it the velocity

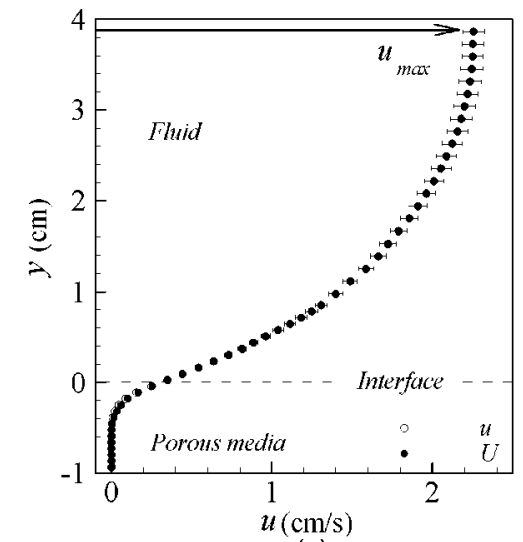

(a)

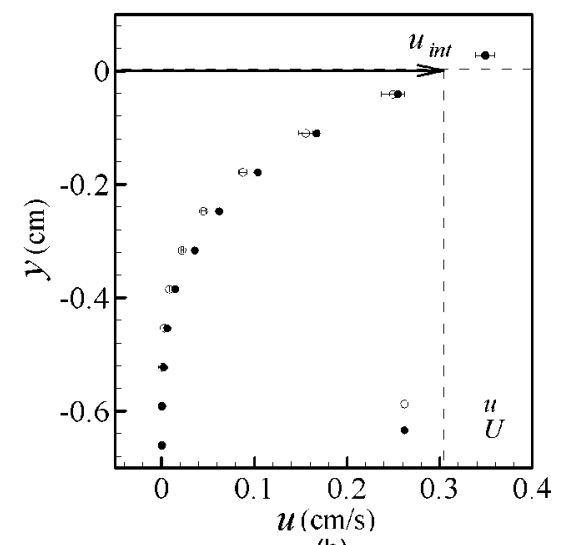

(b)

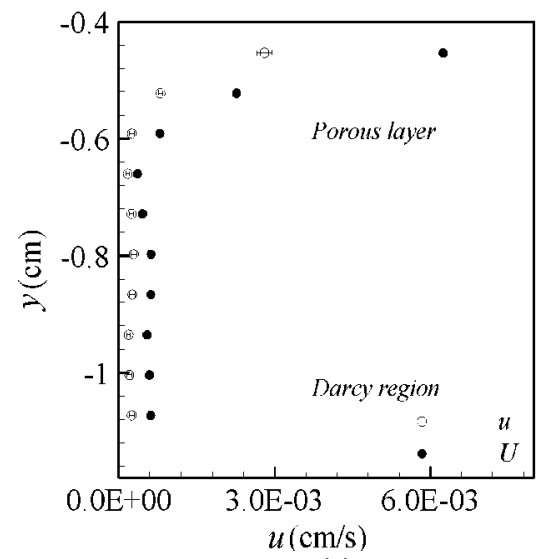

(c)

FIG. 5. Vertical profile of horizontal velocity component: (a) overall plot, (b) zoomed at interfacial region, (c) zoomed inside porous region (GB3, $\left.H_{f}=4 \mathrm{~cm}, \operatorname{Re}_{f}=21\right)$.

fluctuates randomly. All velocity values below the critical point are averaged to $\bar{u}=2.3 \times 10^{-4} \mathrm{~cm} / \mathrm{s}$ with a standard deviation of $\sigma=4.1 \times 10^{-5} \mathrm{~cm} / \mathrm{s}$.

It was found that the deviation of the velocity from its mean value only for all depths below the critical point falls within the errors indicated in the measurements. Hence, the depth of the critical point $y$ can be considered as the lower limit of the transition layer, which amounted to an approximate value of $\delta \sim 0.6 \mathrm{~cm}$ for experiment with the glass beads of diameter $d=0.65 \mathrm{~cm}$.

This hints towards a possible similarity in the order of magnitude of the transition layer thickness and the grain size. 
TABLE V. Magnitudes of the averaged horizontal velocity components at different positions above and below the interface.

\begin{tabular}{cc}
\hline \hline$y(\mathrm{~cm})$ & $u\left(\times 10^{-4} \mathrm{~cm} / \mathrm{s}\right)$ \\
\hline 0.03 & 3480 \\
-0.04 & 2490 \\
-0.11 & 1550 \\
-0.18 & 875 \\
-0.25 & 451 \\
-0.32 & 222 \\
-0.38 & 84 \\
-0.45 & 28 \\
-0.52 & 8 \\
-0.59 & 2.5 \\
-0.66 & 1.7 \\
-0.73 & 2.4 \\
-0.8 & 2.9 \\
-0.87 & 2.6 \\
-0.93 & 1.9 \\
-1.00 & 2 \\
-1.07 & 2.5 \\
\hline \hline
\end{tabular}

In the second method, following the standard definition of the boundary layer thickness, a transition layer thickness may be defined as the distance, measured from the permeable interface $(y=0)$ into the porous medium, at which the velocity first approaches to within, say, $1 \%$ of the Darcy velocity $u_{D}$, namely,

$$
u=1.01 u_{\left.D\right|_{y=-\delta}} .
$$

Employing this equation for the velocity values obtained for our experiments with the aforementioned grain diameter, it was found that condition (6) was satisfied within a depth, approximately equal to $\delta \sim 0.6 \mathrm{~cm}$, which is the same as the order of magnitude of the grain size itself.

As demonstrated, both criteria suggest the same similarity between the order of magnitude of $\delta$ and that of the grain sizes $d$.

Below this limit $(\delta)$, the horizontal velocity component becomes constant with respect to the $y$-direction and corresponds to the well-known Darcy velocity, $u_{D}=\bar{u}=2.3$ $\times 10^{-4} \mathrm{~cm} / \mathrm{s}$. Similar trend was observed with all experiments performed for all other porous samples reported in Table I.

\section{Influence of permeability on the velocity profiles}

To observe the influence of permeability on the velocity profiles, five experiments with different porous samples were performed. The velocity profiles for all samples are plotted in Fig. 6(a). Note that the Reynolds number and fluid height are constant $\left(\operatorname{Re}_{f}=21, H_{f}=4 \mathrm{~cm}\right)$ in this figure. In all subsequent results, only $u$ is presented. As depicted in the figure, the deviation among the velocity profiles increases when approaching the interface. The horizontal velocity component at the permeable interface, $u_{\text {int }}$, increases with permeability and grain size. To demonstrate the influence of permeability

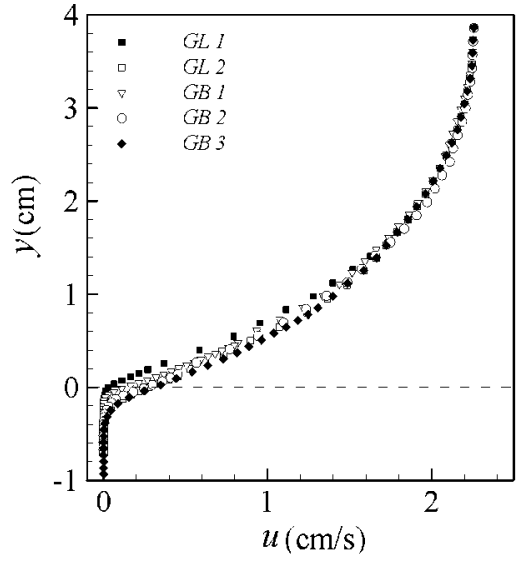

(a)

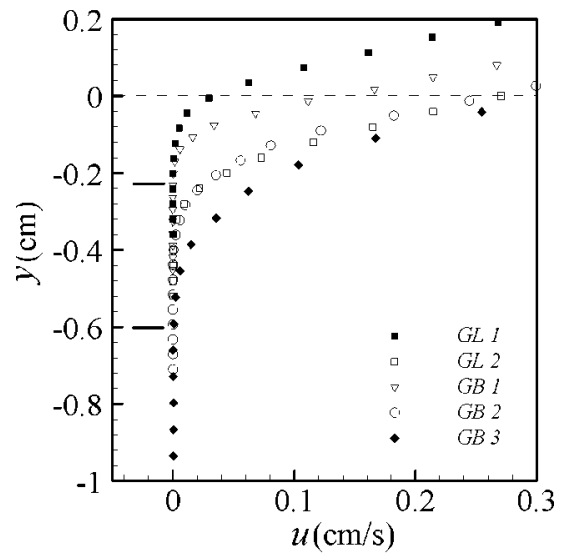

(b)

FIG. 6. Vertical profile of horizontal velocity component for different permeabilities: (a) overall plot; (b) zoomed at the interfacial region $\left(\operatorname{Re}_{f}\right.$ $\left.=21, H_{f}=4 \mathrm{~cm}\right)$.

and the grain size on the thickness of the transition layer, magnified velocity profiles are shown at the interfacial region [Fig. 6(b)].

As can be observed from Fig. 6(b), the thickness of the transition layer, $\delta$, increases with increasing permeability as well as the grain diameter and lies in the range of $0.23 \mathrm{~cm}$ $<\delta<0.6 \mathrm{~cm}$ (denoted by the horizontal bars in the figure). The values of the transition layer thickness for different samples with their corresponding permeabilities and grain diameters are summarized in Table VI.

As shown therein, a trend between the thickness of the transition layer, $\delta$, and the grain diameter $d$ is visible. We will come back to this point below.

\section{Influence of the Reynolds number on the velocity profiles}

To study the response of the interfacial horizontal velocity profile to the Reynolds number $\mathrm{Re}_{f}$ different flow rates were set in the channel by controlling $u_{\max }$ to obtain $\operatorname{Re}_{f}=6$, $10,14,17$, and 21. In this set of experiments the fluid height over the interface and the grain diameter were fixed $\left[H_{f}\right.$ $=4 \mathrm{~cm}, d=0.47 \mathrm{~cm}(\mathrm{~GB} 2)]$. As can be depicted in Fig. 7, the slopes of the velocity profiles depend strongly on $\operatorname{Re}_{f}$ for both regions [see Figs. 7(a) and 7(b)]. Further, Fig. 7(b) 
TABLE VI. Comparison of the transition layer thickness $\delta$ with the square root of permeability $\sqrt{k}$ and the grain size diameter $d$ for different types of granulates and glass beads.

\begin{tabular}{llllll}
\hline \hline & GL1 & GL2 & GB1 & GB2 & GB3 \\
\hline$k\left(\times 10^{-9} \mathrm{~m}^{2}\right)$ & 0.52 & 3.910 & 5.158 & 10.963 & 13.47 \\
$\sqrt{k}\left(\times 10^{-3} \mathrm{~m}\right)$ & 0.023 & 0.0625 & 0.0718 & 0.1047 & 0.116 \\
$d\left(\times 10^{-3} \mathrm{~m}\right)$ & $0.5-2$ & $2.0-3.5$ & 2.5 & 4.7 & 6.5 \\
$\delta\left(\times 10^{-3} \mathrm{~m}\right)$ & 2.4 & 3.6 & 2.3 & 4.3 & 6 \\
\hline \hline
\end{tabular}

shows that $u_{\text {int }}$ increases with $\mathrm{Re}_{f}$, whereas the thickness of the transition layer is not significantly altered.

\section{E. Influence of fluid height on the velocity profiles}

Four different fluid heights $\left(H_{f}=2,3,4,5 \mathrm{~cm}\right)$ over the sediment layer were implemented to study their effects on the transition layer thickness $\delta$. For these experiments, the Reynolds number as well as grain diameter were kept constant $(\operatorname{Re}=21, d=0.47 \mathrm{~cm})$. Note that the constant $\operatorname{Re}_{f}$ was achieved by decreasing $u_{\max }$. The corresponding horizontal velocity profiles are reported in Fig. 8. As can be seen therein, the conclusions one may draw are similar to those given in the preceding section.

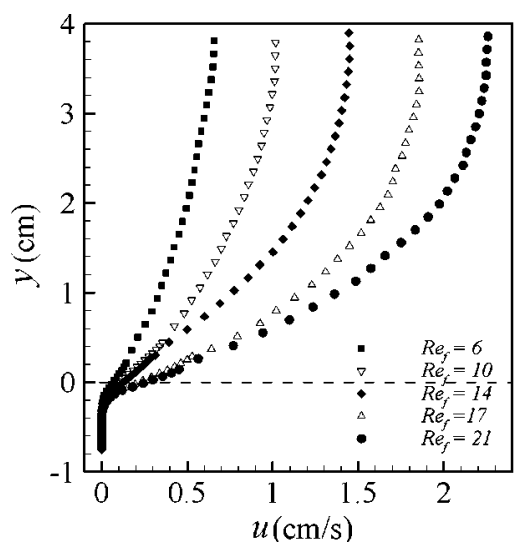

(a)

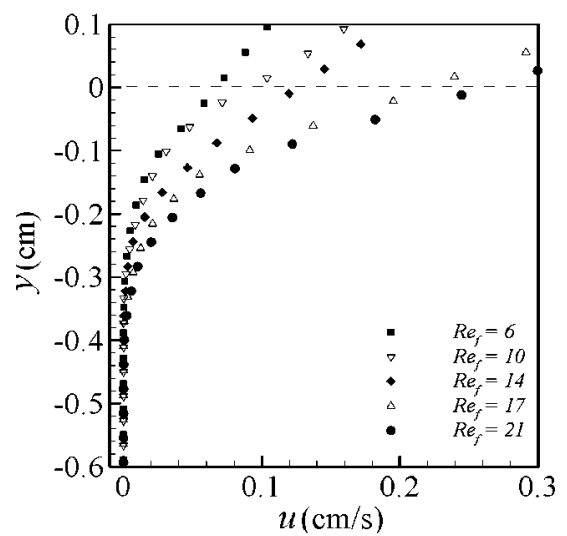

(b)

FIG. 7. Vertical profile of horizontal velocity component for different Reynolds numbers: (a) overall plot; (b) zoomed at the interfacial region (GB2, $\left.H_{f}=4 \mathrm{~cm}\right)$.

\section{F. Correlation between the porosity and velocity profiles}

In order to measure the porosity as a function of depth, the PIV image (Fig. 3) was binarized as follows: the fluid region was set to 0 (white) and the porous area to 1 (black). Then the porosity was determined for each horizontal line given by an ensemble of pixels as the number of pixels denoting the fluid points to the total number of pixels. This was repeated for ten different image sections to obtain an averaged porosity profile (Fig. 9). Far from the interface the porosity is equal to 1 (in the fluid layer). The border of the upper most solid matrix represents the origin of the $y$ coordinate $(y=0)$. Below the origin the porosity decreases linearly until it reaches fluctuating values, which are, in aver-

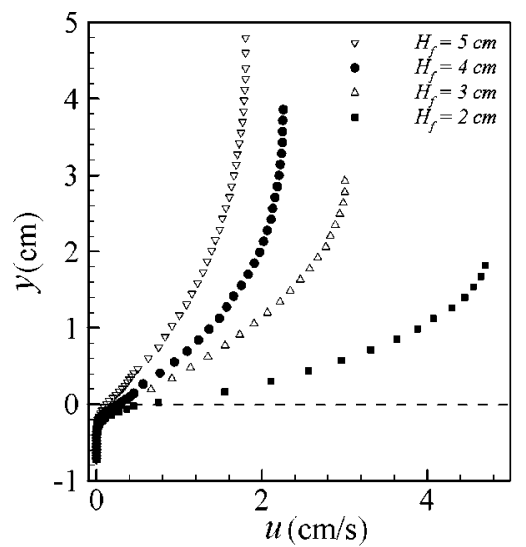

(a)

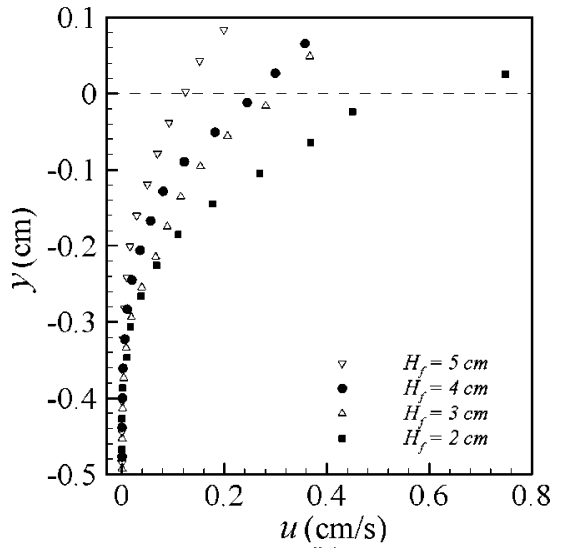

(b)

FIG. 8. Vertical profile of horizontal component velocity vectors for different heights: (a) at fluid and porous media; (b) zoomed at the interfacial region $\left(\mathrm{GB} 2, \mathrm{Re}_{f}=21\right)$. 


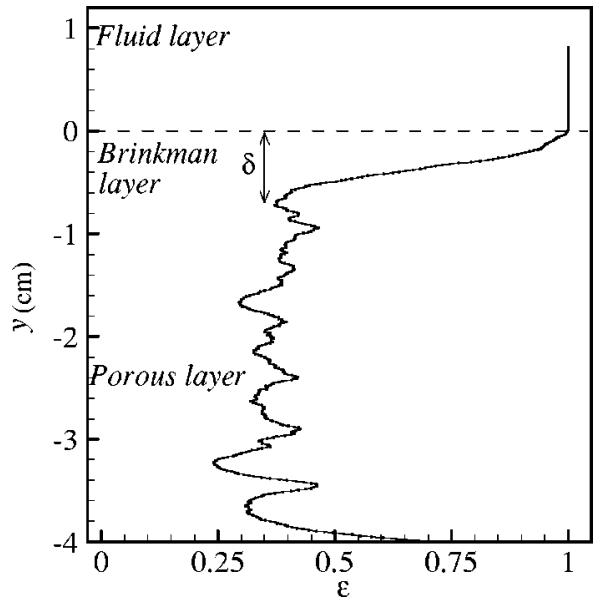

FIG. 9. Porosity variation as a function of depth $\left(\mathrm{GB} 3, H_{f}=4 \mathrm{~cm}\right)$.

age, the same as the bulk porosity $(\varepsilon=0.41)$, measured previously (see Table I). It is interesting to note that the distance between $y=0$ to the depth of the onset of fluctuations was, following the same statistical arguments of Sec. III B, approximately equal to grain size diameter $\sim 0.6 \mathrm{~cm}$. Hence, the order of the magnitude of the porous layer below the interface, in which the porosity linearly decreased was similar to that of the grain size.

\section{DISCUSSION}

\section{A. Length scale of the transition layer}

Since Brinkman's suggestion ${ }^{8}$ for an extension of Darcy's equation, vital discussions ${ }^{4,18-20}$ have been started. Among others, the thickness of the transition layer between the fluid and the pure Darcy region was one of the questions raised, yet without any general consensus and experimental justification. ${ }^{9,21-24}$ Generally, the transition layer is considered to be the vertical extent, in which the flow motion in the fluid layer given by the Navier-Stokes or Stokes equation matches to the Darcy flow in the porous medium.

The length scale of this transition layer has been predicted to be of the order of square root of the permeability, $\sqrt{k}{ }^{6}$ Recently, Gupte and Advani ${ }^{9}$ have investigated the interfacial flow using a Hele-Shaw system, and measured locally a transition layer that exceeded this limit. They concluded that the transition layer thickness was rather comparable to the depth of the Hele-Shaw cell. To study this question in a more general frame, in the present work the interfacial flow between a fluid and porous layer was investigated for different samples, flow rates, and fluid heights over a horizontal porous bed. The transition layer thickness as measured from experiments reported above, is plotted versus $\sqrt{k}$ as well as grain diameter, $d$ [see Figs. 10(a) and 10(b), respectively].

As can be depicted from these figures, relative to grain size we encounter

$$
\frac{\delta}{d}=O(1)
$$

and with respect to $\sqrt{k}$, an average of

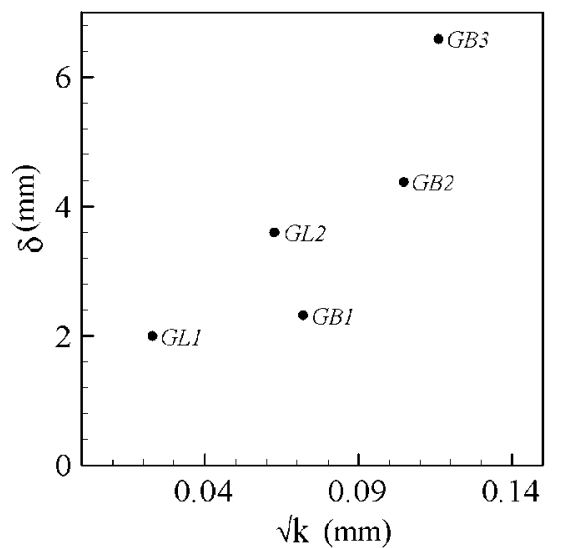

(a)

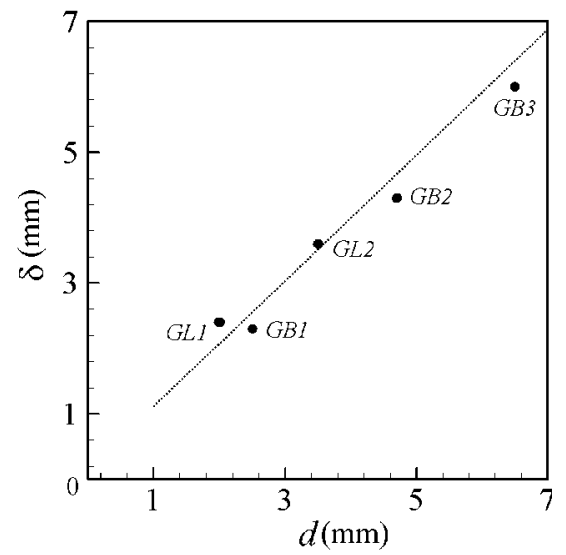

(b)

FIG. 10. The length of the transition zone vs (a) the permeability and (b) the matrix grain size.

$$
\frac{\delta}{\sqrt{k}} \sim O(50)
$$

The first relation, (7), matches with the theoretical investigation of Goyeau et al. ${ }^{24}$ in a one-dimensional flow situation, whereas the second one (8) is comparable to results obtained by Ochoa-Tapia and Whitaker, ${ }^{21,22}$ in which they suggested

$$
\frac{\delta}{\sqrt{k}}=O(30)
$$

Furthermore, considering the Carman-Kozeny relation (see, for example, Nield and Bejan ${ }^{17}$ )

$$
k=\frac{\varepsilon^{3} d^{2}}{180(1-\varepsilon)^{2}}
$$

with the porosities given in Table I, one obtains again a grain diameter of approximately 30 multiplied by $\sqrt{k}$ showing that the experimental relations (7) and (8) are consistent with Carman-Kozeny relation (10), and that some previous theoretical results are confirmed by these experiments.

Therefore, we conclude from our experiments that the transition layer thickness is of the order of the grain diameter rather than the square root of medium permeability. 


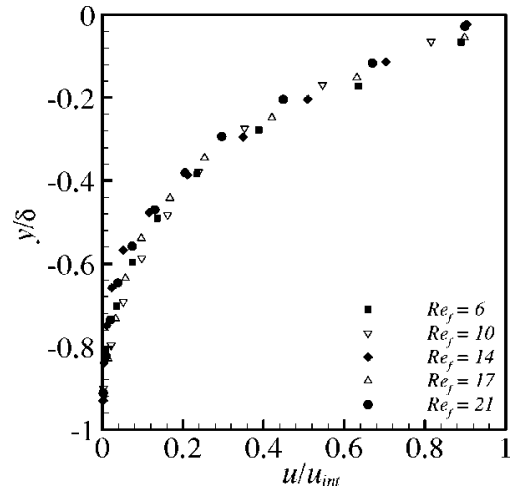

FIG. 11. The nondimensional velocity profile at the transition layer for different Reynolds numbers (GB2, $\left.H_{f}=4 \mathrm{~cm}\right)$.

\section{B. Role of the interfacial velocity}

As discussed previously [Fig. 7(b)], the influence of $\mathrm{Re}_{f}$ on the transition layer thickness is not significant. A similar conclusion was drawn by Choi and Waller, ${ }^{23}$ who stated that the depth of fluid penetration into the porous layer is only dependent on Darcy number Da in the porous media rather than on $\operatorname{Re}_{f}$ or on the shape of the velocity profile.

Although the interfacial velocity gradients for different $\operatorname{Re}$ [shown in Fig. 7(b)] deviate significantly, their nondimensional representation using their corresponding $u_{\text {int }}$ follows almost the same trend for all $\operatorname{Re}_{f}$, when grain diameter is kept constant (here, GB2). This is a clear indication that $u_{\text {int }}$ might be considered as a proper scaling factor for the velocity distribution, as shown in a $y / \delta$ versus $u / u_{\text {int }}$ plot (see Fig. 11).

Furthermore, we investigated whether or not this observation was of a general nature, and could be repeated for other porous samples studied in this paper.

Following the same nondimensional plot, we observed that the same trend seen for GB2 may be observed for other monodisperse glass bead samples (GB1 and GB3) but not for polydisperse granulate samples (GL1 and GL2). Hence, the quantity $u_{\text {int }}$ seems to be a proper scaling factor for monodisperse material, as shown in Fig. 12.

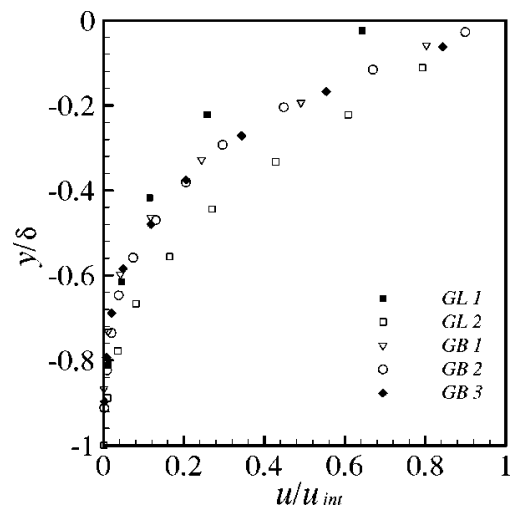

FIG. 12. The nondimensional velocity profile at the transition layer for different permeabilities $\left(H_{f}=4 \mathrm{~cm}, \operatorname{Re}_{f}=21\right)$.

\section{CONCLUSION}

Using RIM and PIV, we investigated a 2D flow field at the interface between a porous matrix and its overlying fluid layer. With this method, fine scale velocity measurements covering small portions of the fluid as well the porous layer were measured and averaged to obtain a single interfacial horizontal velocity profile for different porous samples.

From the averaged velocity profile, it was observed that the horizontal velocity profile decreases continuously when moving downward from fluid into the porous layer. The experimentally obtained data indicate clearly the existence of a transition layer, which is characterized by a drastic decrease of velocity as a matching zone between the pure fluid and the Darcy region. The length scale of the transition layer was found to be of the order of grain diameter and much larger than the square root of permeability. The vertical gradient of the velocity depends strongly on the Reynolds number and the height of the fluid layer. Furthermore, it was observed that the interfacial velocity represents a proper scaling factor only for a monodisperse porous matrix. The flow field inside polydisperse media that are more closely related to natural situations needs further detailed studies in the future.

\section{ACKNOWLEDGMENTS}

This study was supported by the Deutsche Forschungsgemeinschaft (DFG). The authors are grateful to Dow Corning $\mathrm{GmbH}$ for providing Dow Corning 550 and Dow Corning 556 free of cost. A.K. is grateful to D. A. Nield for his valuable comments and corrections before submission of the manuscript, and to two anonymous referees for their valuable comments.

${ }^{1}$ H. C. Brinkman, "A calculation of the viscous force exerted by a flowing fluid on a dense swarm of particles," Appl. Sci. Res., Sect. A 1, 27 (1947).

${ }^{2}$ G. Neale and W. Nader, "Practical significance of Brinkman's extension of Darcy's law: Coupled parallel flows within a channel and a bounding porous medium," Can. J. Chem. Eng. 52, 475 (1974).

${ }^{3}$ T. Levy and E. Sanchez-Palencia, "On boundary conditions for fluid flow in porous media," Int. J. Eng. Sci. 13, 923 (1975).

${ }^{4}$ T. S. Lundgren, "Slow flow through stationary random beds and suspensions of spheres," J. Fluid Mech. 51, 273 (1972).

${ }^{5} \mathrm{~K}$. Vafai and R. Thiyagaraja, "Analysis of the flow and heat tranfer at the interface region of a porous medium," Int. J. Heat Mass Transfer 30, 1391 (1987).

${ }^{6}$ M. Kaviany, Principles of Heat Transfer in Porous Media (Springer, New York, 1995).

${ }^{7}$ B. B. Jørgensen, "Life in the diffusive boundary layer," in The Benthic Boundary Layer: Transport Processes and Biogeochemistry, edited by B. P. Boudreau and B. B. Jørgensen (Oxford University Press, London, 2001), pp. 348-373.

${ }^{8} \mathrm{H}$. C. Brinkman, "On the permeability of media consisting of closely packed porous particles," Appl. Sci. Res., Sect. A 1, 81 (1947).

${ }^{9}$ S. K. Gupte and S. G. Advani, "Flow near the permeable boundary of porous medium: An experimental investigation using lda," Exp. Fluids 22, 408 (1997).

${ }^{10}$ A. Khalili, A. J. Basu, and U. Pietrzyk, "Flow visualization in porous media via positron emission tomography," Phys. Fluids 10, 1031 (1998).

${ }^{11}$ C. H. Liu, C. Vafidis, and J. H. Whitelaw, "Flow in the coolant passages of an internal combustion engine cylinder head," Exp. Fluids 10, 50 (1990).

${ }^{12}$ M. Rashidi, L. Peurrung, A. Tompson, and T. Kulp, "Experimental analysis of pore-scale flow and transport in porous media," Adv. Water Resour. 19, 163 (1996).

${ }^{13} \mathrm{~A}$. Cenedese and P. Viotti, "Lagrangian analysis of nonreactive pollutant dispersion in porous media by means of the particle image velocimetry technique," Water Resour. Res. 17, 2329 (1996). 
${ }^{14}$ M. A. Northrup, T. J. Kulp, and S. M. Angel, "Fluorescent particle image velocimetry-Application to flow measurement in refractive indexmatched porous-media," Appl. Opt. 30, 3034 (1991).

${ }^{15}$ R. C. Givler and S. A. Altobelli, "A determination of the effective viscosity for the Brinkman-Forchheimer flow model," J. Fluid Mech. 258, 355 (1994).

${ }^{16}$ J. C. Ward, "Turbulent flow in porous media," J. Hydraul. Div., Am. Soc. Civ. Eng. 90, 1 (1964).

${ }^{17}$ D. A. Nield and A. Bejan, Convection in Porous Media (Springer, New York, 1999).

${ }^{18}$ C. K. W. Tam, "The drag on a cloud of spherical particles in low Reynolds number flow," J. Fluid Mech. 38, 537 (1969).

${ }^{19}$ D. A. Nield, "The boundary correction for Rayleigh-Darcy problem: limitations of the Brinkman equation,” J. Fluid Mech. 128, 37 (1983).
${ }^{20}$ L. Durlofsky and J. F. Brady, "Analysis of the Brinkman equation as a model for flow in porous media," Phys. Fluids 30, 3329 (1987).

${ }^{21}$ J. A. Ochoa-Tapia and S. Whitaker, "Momentum transfer at the boundary between a porous medium and a homogeneous fluid-I. Theoretical development," Int. J. Heat Mass Transfer 38, 2635 (1995).

${ }^{22}$ J. A. Ochoa-Tapia and S. Whitaker, "Momentum transfer at the boundary between a porous medium and a homogeneous fluid-II. Comparison with experiment," Int. J. Heat Mass Transfer 38, 2647 (1995).

${ }^{23}$ C. Y. Choi and P. M. Waller, "Momentum transport mechanism for water flow over porous media," J. Environ. Eng. 123, 792 (1997).

${ }^{24}$ B. Goyeau, D. Lhuillier, D. Gobin, and M. G. Velarde, "Momentum transport at a fluid-porous interface," Int. J. Heat Mass Transfer 46, 4071 (2003). 